\title{
Introduction au dossier - Faire école en contexte de pandémie
}

Entre mars et juin 2020, la très vaste majorité des pays ont fermé leurs établissements préscolaires, primaires et secondaires à cause de la pandémie causée par la Covid-19. Selon l'UNESCO, près de deux milliards d'élèves et environ 60 millions d'enseignants ont été directement concernés par cette mesure, qui est unique par son ampleur dans l'histoire moderne de l'éducation depuis le 17e siècle. En août et septembre 2020, alors que la première vague de la pandémie semblait avoir ralenti, certains pays ont commencé à ouvrir leurs écoles, souvent selon des formules hybrides alternant enseignement en classe et enseignement à distance. Toutefois, dès septembre 2020, une seconde vague de la Covid-19 a pris énormément d'ampleur, forçant les gouvernements à improviser et à multiplier les stratégies; écoles fermées, écoles ouvertes à mi-temps, écoles ne fonctionnant qu'avec une partie des élèves, écoles utilisant diverses formules hybrides, etc. Peu importe le choix ou les choix retenus, il est clair que les écoles ne peuvent pas du tout fonctionner normalement à cause des mesures de distanciations sociales et des risques, constants, d'une amplification potentiellement catastrophique de la pandémie pour les sociétés et leurs économies.

Depuis septembre 2020, on observe à peu près partout combien il est difficile, aussi bien pour les élèves que pour les enseignants, de reprendre le chemin de l'école, alors que les établissements scolaires ne fonctionnent souvent qu'à moitié de leurs effectifs, tandis que les mesures sanitaires les obligent à réduire au strict minimum tous les déplacements et les contacts entre élèves les enseignants. En même temps, les autorités politiques et éducatives exigent de ces derniers qu'ils mettent rapidement en place des pratiques pédagogiques avec lesquelles beaucoup d'entre eux semblent encore peu familiers: enseignement à distance, entretien téléphonique avec leurs élèves, voire leurs parents, travaux à réaliser à la maison, rencontres à l'extérieur de l'école, etc. Or, ces pratiques semblent rencontrer rapidement leurs 
limites lorsqu'elles sont mises en œuvre auprès des jeunes élèves du préscolaire et du primaire, des élèves en difficulté et des élèves des régions et quartiers défavorisés. De manière générale, il semble maintenant évident que la crise actuelle touche plus durement ces groupes d'élèves, ce qui risque d'accentuer les inégalités déjà largement présentes au sein des systèmes d'enseignement

La présente situation soulève au fond une interrogation fondamentale : comment peut-on enseigner et apprendre aujourd'hui hors de l'institution scolaire ou du moins hors de son organisation traditionnelle, c'est-à-dire des classes fermées regroupant habituellement entre 20 et 40 élèves sous la gouverne d'un enseignant qui enseigne la même chose à tous toute la journée? Cette organisation a été conçue et mise en place dès le début du 17e siècle en Europe par les communautés religieuses enseignantes. Elle s'est répandue au 19e siècle et surtout au 20e siècle avec la formidable expansion et l'allongement de l'école obligatoire. Elle est aujourd'hui le modèle dominant, pour ne pas dire unique de l'éducation des nouvelles générations.

À l'heure actuelle, la plupart des personnes qui réfléchissent à la question précédente cherchent avant tout à temporiser, en espérant un éventuel retour à la normale du monde scolaire. Temporiser, c'est vouloir gagner du temps, en cherchant des moyens du suppléer temporairement à la fermeture des écoles ou à leurs difficultés de fonctionnement une fois qu'elles sont ouvertes. Mais on peut aussi pousser cette réflexion plus loin, en s'interrogeant sur des pratiques pédagogiques et organisationnelles qui pourraient éventuellement contribuer à définir d'autres formes de scolarisation des enfants et des jeunes, c'est-à-dire d'autres manières d'enseigner et d'apprendre hors de l'école ou hors de son fonctionnement traditionnel.

Lobjectif de ce numéro hors-série est de convoquer ces deux types de réflexion, en faisant appel à des expertises de recherche et à des expertises pédagogiques sur les différentes manières d'enseigner et d'apprendre, soit dans une école en attente d'un retour à sa normalité, soit dans une école cherchant à se réinventer, en repensant de manière plus radicale ses pratiques traditionnelles.

Les textes présentés dans ce dossier hors-série de la revue internationale Formation et profession portent tous sur l'enseignement ou l'apprentissage en contexte de pandémie. Leurs auteurs proviennent du Canada, de la France, de Belgique, de la Suisse, de la Russie, de la Côte d'Ivoire et des Antilles. Rédigés par des chercheurs et/ou des praticiens, ils visent à documenter, par la réflexion, par la recherche ou par des retours d'expérience, la mise en place de pratiques enseignantes susceptibles de soutenir l'apprentissage des élèves dans et hors l'école. Du même coup, ce numéro hors-série entend offrir aux acteurs éducatifs des voies d'exploration, pour mieux repenser la pédagogie scolaire et l'adapter à la crise actuelle, tout en anticipant des formes et des pratiques nouvelles pour enseigner et apprendre, formes et pratiques fécondantes et porteuses d'avenir pour la pédagogie de demain.

\section{Pour citer cet article}

Borges, C., Tardif, M. et Karsenti, T. (2020). Introduction au dossier : Faire école en contexte de pandémie. Formation et profession, 28(4 hors-série), 1-2. http://dx.doi.org/10.18162/fp.2020.684

2 - Formation et profession $28(4$ hors-série), 2020 